\title{
Joint framework with deep feature distillation and adaptive focal loss for weakly supervised audio tagging and acoustic event detection
}

\author{
Yunhao Liang ${ }^{\mathrm{a}}$, Yanhua Long, ${ }^{\mathrm{a}, *},{\text { Yijie } \mathrm{Li}^{\mathrm{b}} \text {, Jiaen Liang }}^{\mathrm{b}}$, Yuping Wang ${ }^{\mathrm{c}}$ \\ ${ }^{a}$ Key Innovation Group of Digital Humanities Resource and Research, \\ Shanghai Normal University, Shanghai, China \\ ${ }^{b}$ Unisound AI Technology Co., Ltd., Beijing, China \\ ${ }^{c}$ ByteDance, Beijing, China
}

\begin{abstract}
A good joint training framework is very helpful to improve the performances of weakly supervised audio tagging (AT) and acoustic event detection (AED) simultaneously. In this study, we propose three methods to improve the best teacher-student framework in the IEEE AASP Challenge on Detection and Classification of Acoustic Scenes and Events (DCASE) 2019 Task 4 for both audio tagging and acoustic events detection tasks. A frame-level target-events based deep feature distillation is first proposed, which aims to leverage the potential of limited strong-labeled data in weakly supervised framework to learn better intermediate feature maps. Then, we propose an adaptive focal loss and two-stage training strategy to enable an effective and more accurate model training, where the contribution of hard and easy acoustic events to the total cost function can be automatically adjusted. Furthermore, an event-specific post processing is designed to improve the prediction of target event time-stamps. Our experiments are performed on the public DCASE 2019 Task 4 dataset, results show that our approach achieves competitive performances in both AT (81.2\% F1-score) and AED (49.8\% F1-score) tasks.
\end{abstract}

Keywords: Acoustic event detection, feature distillation, adaptive focal loss, event-specific post processing

\section{Introduction}

Audio tagging (AT) refers to predict the category of acoustic events that occur in an audio segment, while the task of acoustic event detection (AED) not only needs to identify whether certain events occur in this segment, but also

\footnotetext{
${ }^{*}$ Corresponding author

Email address: yanhua@shnu.edu.cn (Yanhua Long )
}

Preprint submitted to Digital Signal Processing

February 15, 2022 
needs to detect their onset and offset time-stamps. The AED and AT technology can be applied in many areas, such as smart home [1, health monitoring systems [2] and multimedia retrieval [3, 4], etc. Because labeling large-scale training data with detailed annotations is high cost and time-consuming, the challenges of Detection and Classification of Acoustic Scenes and Events (DCASE) from 2018 to 2020 Task 4 [5, 6, 7] have been launched, to explore the possibility for exploiting a large amount of unbalanced and unlabeled training data together with a small weakly annotated training set to improve AED system performance. These challenges have attracted increasing research attention in this field [8, 9, 10, 11. In this study, we also focus on this weakly supervised task to improve both of the AT and AED system performances.

In the literature, many previous works have been proposed to improve the AED systems from variety aspects, such as proposing new back-end classifiers, new robust features, better post-processing methods, and training data enhancement techniques, etc. For the back-end classifiers, [12] proposed to use a specially designed convolutional neural networks instead of the traditional HMM and SVM to exploit a novel spectrogram image feature for improving system performance. In [13, authors proposed a gated multi-head attention pooling algorithm, to attend the information of events from different heads at different positions. Work in [14 proposed a new classifier that consists of a modified DenseNet as the feature extractor, and a global average pooling layer to predict the frame-level labels at inference time. This classifier can directly localize target events using the feature map that extracted by DenseNet without any recurrent layers. Authors in [15] proposed a two-stage polyphonic SED model to handle the sound events overlapping in time-frequency. A faster regional CNN and an attention-LSTM were used in the first stage to capture those region-ofinterests. In the second stage, two CNNs combined with one LSTM were used for further feature representation, the followed softmax combined with CTC was then used for the final event detection and classification. As mentioned above, most of those previously proposed back-end classifiers focused on exploiting the discriminative information in different-level feature representations.

At the feature level, most previous works focused on investigating better robust feature learning techniques, such as in [16, 17, a Multi-Scale TimeFrequency Attention (MTFA) module to enhance the acoustic features for AED was proposed. It gathers information at multiple resolutions to generate a timefrequency attention mask which tells the model where to focus along both time and frequency axis. With MTFA, the model could capture the characteristics of target events with different scales. They achieved competitive results on DCASE 2017 Task 2. In [18, authors focused on proposing new discriminative feature learning method, they aimed to reduce the intra-class sample distances and increase inter-class sample distances rather than estimating the pair-wise distances of samples to learn better class discrimination at feature-level. By adding a distance metric constraint on feature extraction, the learned features could have a good property for improving the generalization of the classification models. And works in [19] also focused on exploring robust features for different sound classification tasks, they introduced a multi-stream convolutional neural 
network with temporal attention, to learn a high-level robust feature representation from three input streams, including the raw audio, the spectral features and the temporal attention-based energy features.

During inference, post-processing is applied to smooth each event-class probability sequence. In recent works, some better post-processing methods has been proposed. Such as, in [20, the impact of fixed-size window median filtering was investigated, and they proposed a double thresholding as a more robust and predictable post-processing method to improve the DCASE 2018 Task 4 AED performances. And in [21, three post-processing methods have been proposed for smoothing the neural network outputs: the median filtering, accept gap, and removal of the short duration events.

In addition, variety of data augmentation techniques are used in AED to improve the generalization ability of models. For example, 22] perturbed the audio inputs by mixing in other environmental audio clips, and leverage other training examples as sources of noise. [23] used random time and frequency shifts as natural perturbations on audio data. 24 proposed a mixup augmentation to train the neural network using convex combination of pairs of samples. In [25, authors further extended this mixup to image detection tasks to provide a good consistency perturbation for semi-supervised learning.

Looking at the recent approaches surveyed above, we see all of the backend classifiers, features, training data augmentation and post-processing affect final AED and AT system performances. However, for the weakly supervised AED tasks, the key issue is how to design a better modeling framework to fully exploit the large-scale weakly and unlabeled training data. Therefore, many recent works focus on the system modeling framework, semi-supervised or unsupervised learning approaches, such as, the joint training system in [26, 27. In DCASE 2018 Task 4, the baseline used two convolutional recurrent neural network (CRNN) with a two-pass training strategy to predict labels of unlabeled clips [28; The best system of this challenge was based on the Meanteacher (MT) model [29, 30, in which two same CRNN networks were taken as the teacher and student model with weight-averaged consistency targets to exploit the large amount of unlabeled data; In DCASE 2019 Task 4, the Guided Learning (GL) model 31 convolutional system achieved the best results. The GL is also a teacher-student (T-S) framework, but unlike the homologous T-S model in MT, GL uses two different model structures to enable an appropriate trade-off between AT and AED. These two models are trained synchronously and forced to learn from the unlabeled data with tags generated by each other.

In this study, our work is also based on the Guided Learning architecture. The contributions are: 1) we modify the GL to learn the models for AED and AT as two independent branches in a joint framework; 2) a frame-level targetevents based deep feature distillation is proposed, and it aims to leverage the maximum potential of limited strong-labeled data; 3) to enable an effective and more accurate model training, a two-stage training strategy with an adaptive focal loss is further investigated; 4) an event-specific post processing is specially designed to fix the prediction errors that result from outliers. It is worth noting that, during the two-stage training, the contribution of hard (difficult- 
to-classify) and easy (easy-to-classify) acoustic events to the total cost function will be dynamically adjusted in each iteration, which makes the importance of easy events are down-weighted and the model can rapidly focus on hard events in later stages. Experimental results on the DCASE 2019 Task 4 challenge show that our proposed methods can achieve competitive performances in both AT and AED tasks.

The rest of the work is organised as follows. In Section 2, we introduce our proposed methods, including the AD and AED joint framework in Section 2.1. the target-events based deep feature distillation (TFD) in Section 2.2 the twostage training with adaptive focal loss in Section 2.3. and the event-specific post processing (ESP) in Section 2.4. Section 3 presents the experiments, results and analysis in detail. Finally, this work is concluded in Section 4.

\section{Proposed method}

In this section, we introduce our proposed methods on AED and AT tasks, mainly focusing on the joint framework in Figure 1 . The whole proposed framework is first introduced in section 2.1. followed by the target-events based deep feature distillation (TFD) in section 2.2 the two-stage training with adaptive focal loss in section 2.3. and in section 2.4 we present the details of event-specific post processing (ESP).

\subsection{Joint framework}

The proposed joint architecture for both weakly supervised AT and AED is shown in Figure 1. It consists of four parts: the (a) teacher model, (b) student model, (c) deep feature distillation module and (d) event-specific post processing module. The teacher and student models are Convolution Recurrent Neural Networks (CRNNs), but with different number of CNN blocks. The teacher model has five double-layer CNN blocks with a larger time compression scale that professional for a better audio tagging, while the student model only has three single-layer CNN blocks with no temporal compression scale for a better event boundary detection. Compared with the two same networks structure in conventional Mean-teacher model [29], our smaller student model can not only learn different feature information, but also can reduce the model parameters and improve the training efficiency. Figure 2 shows the detail structural of each single CNN block that included in both teacher and student models. It is composed of a convolutional layer, batch normalization and a ReLU activation function.

Compared with the Guided Learning model in 31] that is only composed of two different convolution networks (CNN Blocks in (a) and (b)), we propose to add two additional bi-directional Gated Recurrent Unit (BGRU) layers after the CNN blocks for extracting the temporal information of CNN representations for a better audio tagging. Different from GL and CRNN frameworks, we first separate the AED and AT tasks into two independent branches as shown in (a) and (b). The AED branch uses a fully connected layer with larger hidden states 


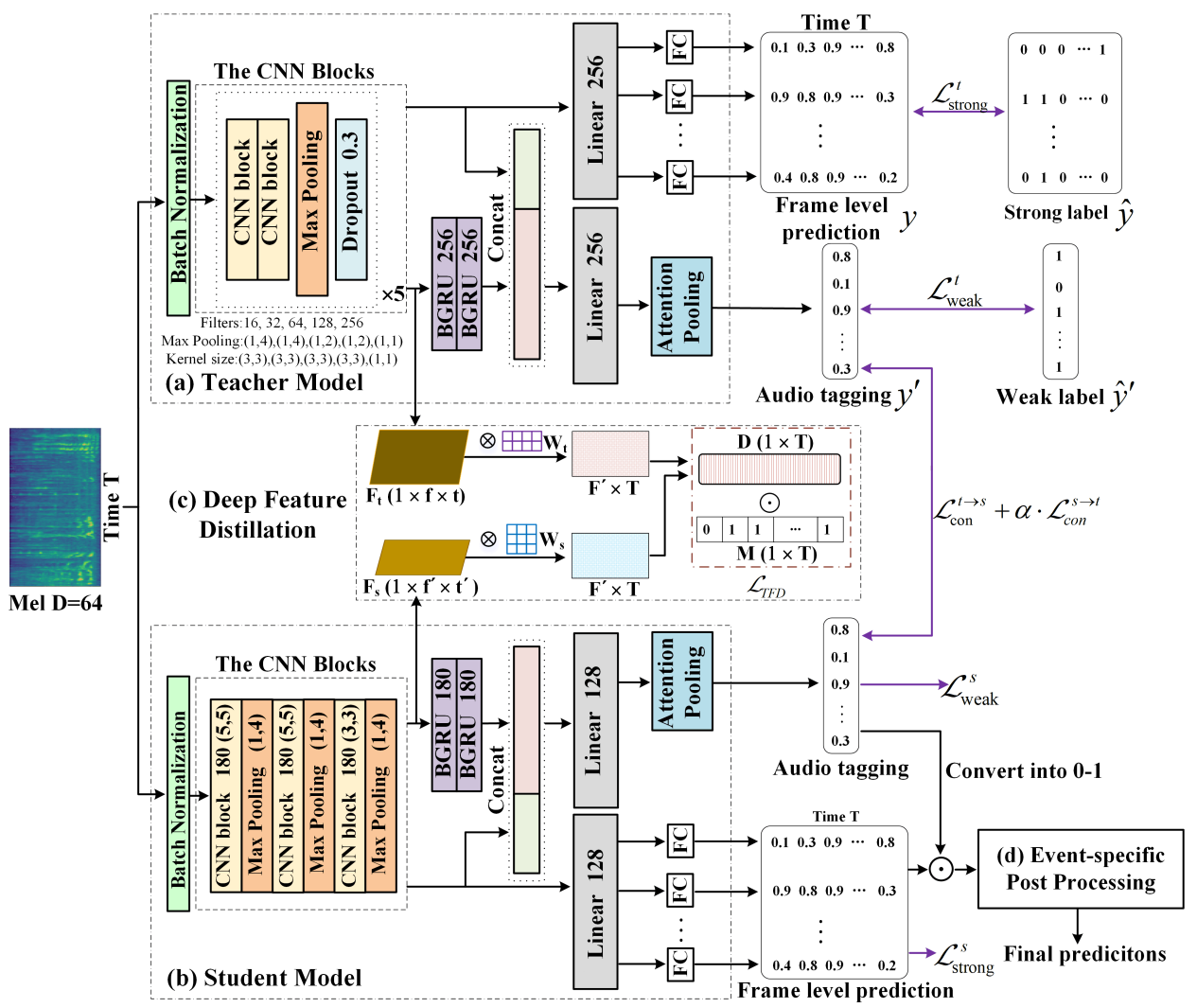

Figure 1: Model architecture of the proposed joint framework for both audio tagging and acoustic event detection.

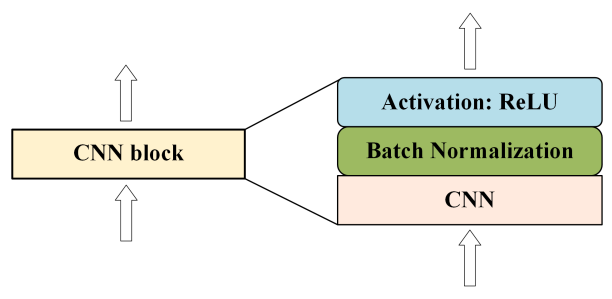

Figure 2: Structure of a single CNN block. 
(the 'Linear' block) followed by ten small size separate fully connected layers (FC) with sigmoid activation to model the outputs of CNN blocks for detection. While in the AT branch, the outputs of both CNN Blocks and BGRUs are concatenated as the input features of 'Linear' layer, followed by an attention pooling module [30] with a consistency loss to perform the events classification. The motivation of this proposal is that, the design of AED system structure requires to accurately reflect the frame-level time-stamps information for detection, and it trains on strongly labeled data, while the AT system does not. Thus, by sharing the same standard feature encoder of CNN Blocks, separate AT and AED into two independent branches can provide possibility for improving the two different tasks independently, such as the two additional BGRU layers designed for the AT branch. The temporal feature representations produced by BGRUs can capture robust sequential information in the audio, which can provide significant complementary information to the standard feature representations from CNN Blocks.

Besides the independent AED and AT branch, we also propose a deep feature distillation in module (c), where only the frame-level feature representations of target-events are used to compute the distillation loss, which can maximally leverage the information of limited strongly labeled data to regulate the teacherstudent model training. Moreover, a two-stage model training strategy with adaptive focal loss is investigated, it can automatically exploit the importance of hard training acoustic events and achieve more stable models. Finally, an event-specific post processing (module $(\mathrm{d})$ ) is proposed to further improve the accuracy of final predictions. In addition, as shown in the block between student model and event-specific post processing, we apply an element-wise multiplication between the one-hot audio tagging prediction labels and the frame-level detection posteriors to ensure the consistency of detection and audio classification results.

\subsection{Target-events based deep feature distillation (TFD)}

Knowledge distillation (KD) is one popular technique that used in machine learning to improve a smaller student model given large teacher model guiding [32, 33, 34. It can be performed not only on the output logits [35, 36] between teacher and student model, but also on activations, neurons, or intermediate features [37, 38, 39]. For the feature-based knowledge distillation, most related works choose to distill the intermediate information between teacher and student model using the whole feature maps directly, including the intra-utterance similarity preserving knowledge distillation that proposed in [40] for audio tagging, the similarity matrix is also calculated on the whole feature maps.

In this study, our goal is to improve the target-events boundary detection performance in weakly supervised AED task, how to maximally exploit the frame-level alignment information of the available limited strong-labeled data is crucial. Therefore, instead of using the whole feature maps to perform the knowledge distillation, we propose to force the distillation performed only on the frame-level deep feature vectors that belong to target-events. The specific 
operations are illustrated in module (c) of Figure 1 and we define the distillation loss function as,

$$
\mathcal{L}_{T F D}=\frac{1}{T}((\mathbf{D} \odot \mathbf{M}) \cdot \mathbf{A})
$$

where $\mathbf{M} \in \mathbb{R}^{1 \times T}$ is the frame-level label mask vector with $j$-th element $\mathbf{M}_{j} \in$ $[0,1]$, and 1 means $j$-th frame is labeled as one type of target events in the limited strong-labeled dataset. $\mathbf{A}=[1,1, \ldots, 1]_{T \times 1}^{\top}, T$ is the frame size, $\odot$ and $\cdot$ denote the element-wise and normal matrix multiplication respectively. $\mathbf{D} \in \mathbb{R}^{1 \times T}$ is a frame-level similarity matrix, where $\mathbf{D}_{j}, j=1,2, \ldots, T$ represents $j$-th frame Euclidean distance between the transformed deep feature vectors of CNN blocks outputs in teacher and student model, and it is defined as,

$$
\mathbf{D}_{j}=\left\|\left(\mathbf{F}_{t} \cdot \mathbf{W}_{t}\right)_{j}-\left(\mathbf{F}_{s} \cdot \mathbf{W}_{s}\right)_{j}\right\|_{2}
$$

where $\left(\mathbf{F}_{t} \cdot \mathbf{W}_{t}\right)_{j}$ and $\left(\mathbf{F}_{s} \cdot \mathbf{W}_{s}\right)_{j}$ is $j$-th column of transformed feature maps $\mathbf{F}_{t}$ and $\mathbf{F}_{s}$ respectively, $\mathbf{W}_{t}$ and $\mathbf{W}_{s}$ are their transformation matrix as shown in module (c) of Figure 1.

By introducing the distillation loss $\mathcal{L}_{T F D}$ into the total training loss of weakly supervised framework, we hope it can maximally leverage the information of limited strong-labeled data to regulate the teacher-student model training, especially for guiding the early training stages of T-S model, because all the information captured by the $\mathcal{L}_{T F D}$ is derived from those target-events with golden-standard time-stamps.

Different from the proposed TFD, similar to [40, most conventional deep feature distillation works perform the deep feature KD directly on the whole transformed maps $\left(\mathbf{F}_{t} \cdot \mathbf{W}_{t}\right),\left(\mathbf{F}_{s} \cdot \mathbf{W}_{s}\right)$ using the conventional Mean Squared Error (MSE) as similarity measure (DFD-MSE), or the frame-level Euclidean distance similarity matrix $D$ (DFD-D). The advantage of DFD-MSE and DFD$\mathrm{D}$ is that, they can use all the available strongly-labeled, weakly and unlabeled training data for the knowledge distillation, instead of the limited stronglylabeled data in our proposed TFD. Experimental comparisons are presented in Section 3.3 .

\subsection{Two-stage training with adaptive focal loss}

Under the weakly supervised AED task like DCASE 2019 Task 4, three types of dataset with different level of annotations are provided, i.e., a limited strong labeled set with time-stamps for target acoustic events, a small weakly annotated set with only the multiple events presence labels (without time-stamps), and a large amount of unlabeled training dataset. To make full use of all the available datasets, recent work in [31] proposed a Guided Learning (GL) strategy, in which the total connectional binary cross entropy (BCE) loss of the weakly supervised T-S training consists of two parts as follows:

$$
\mathcal{L}=\mathcal{L}_{\text {weak }}^{t, s}+\mathcal{L}_{\text {strong }}^{t, s}+\mathcal{L}_{\text {con }}^{t \rightarrow s}+\alpha \cdot \mathcal{L}_{\text {con }}^{s \rightarrow t}
$$

where $\mathcal{L}_{\text {weak }}^{t, s}=\mathcal{L}_{\text {weak }}^{t}+\mathcal{L}_{\text {weak }}^{s}, \mathcal{L}_{\text {strong }}^{t, s}=\mathcal{L}_{\text {strong }}^{t}+\mathcal{L}_{\text {strong }}^{s}$ are the clip-level and frame-level supervised loss as shown in Figure 1 for the AT and AED 
respectively. The last two terms are the clip-level consistency losses performed on all types of training dataset. $\mathcal{L}_{\text {con }}^{t \rightarrow s}$ denotes using teacher predictions to guide the student model training, while $\mathcal{L}_{\text {con }}^{s \rightarrow t}$ denotes using the student to fine-tune the teacher model with a small weight $\alpha$. During the earlier T-S training, only the first three terms are used, the last term is normally added when the teacher model becomes relatively stable. More details can be found in 29, 31. In Equation (3) and the following Equation (6), all the loss with 'weak' subscript are computed using the weakly labeled training data, while the loss with 'strong' and 'con' subscripts are computed using the strongly labeled and all types of training data, including the strongly, weakly and unlabeled parts, respectively.

Motivated by the principle of focal loss in 41, here we aim to improve the GL training by combining the above BCE loss with an adaptive focal loss that is defined as follows:

$$
\mathcal{L}_{a f}=-\frac{1}{C K} \sum_{j=1}^{C} \sum_{i=1}^{K}\left(1-p_{i j}^{\gamma}\right) \cdot \log \left(p_{i j}\right)
$$

where $\gamma$ is a scaling factor to control the loss contribution of posterior probability $p_{i j}$ for $i$-th clip, $j$-th target-event category. $K$ is the total size of audio clips with both weakly and strong labels in a minbatch, $C$ is the number of target-event categories. Figure 3 illustrates the distribution of class-wise weight in the $\mathcal{L}_{a f}$ that varies with its corresponding posterior probability at one training epoch. In Figure 3 , the left Y-axis represents the class-wise posterior probability $p_{j}=$ $\sum_{i=1}^{K} p_{i j}$, and the right $\mathrm{Y}$-axis represents the corresponding class-wise adaptive weights $W_{p_{j}}=\sum_{i=1}^{K}\left(1-p_{i j}{ }^{\gamma}\right)$. From Figure 3 , it's clear to see that the $\mathcal{L}_{a f}$ tends to assign a higher weight to the hard (small $p_{i j}$ ) events than the easy ones, it automatically forces the model pays more attention to the learning of this hard category in next training iteration, and it is dynamically adapted in each training epoch.

Moreover, to guarantee the basic ability of BCE loss for achieving a relatively stable T-S model, we find that the adaptive focal loss should be utilized in a curriculum learning 42 way. Therefore, we propose a two-stage training strategy to train the whole teacher-student model of Figure 1 as follows:

Stage 1, performing the normal GL training together with the proposed TFD in first $\tau$ epochs using:

$$
\mathcal{L}_{s 1}=\mathcal{L}+\beta \cdot \mathcal{L}_{T F D}
$$

where $\beta \in[0,1]$ is used to control the distillation depth.

Stage 2, performing the fine-tuning epochs by replacing the $\mathcal{L}_{\text {weak }}^{t, s}, \mathcal{L}_{\text {strong }}^{t, s}$ in $\mathcal{L}$ using their corresponding adaptive focal loss $\mathcal{L}_{\text {af-weak }}^{t, s}, \mathcal{L}_{\text {af-strong }}^{t, s}$ respectively as,

$$
\begin{gathered}
\mathcal{L}_{s 2}=\mathcal{L}^{\prime}+\beta \cdot \mathcal{L}_{T F D} \\
\mathcal{L}^{\prime}=\mathcal{L}_{\text {af-weak }}^{t, s}+\mathcal{L}_{\text {af-strong }}^{t, s}+\mathcal{L}_{\text {con }}^{t \rightarrow s}+\alpha \cdot \mathcal{L}_{\text {con }}^{s \rightarrow t}
\end{gathered}
$$




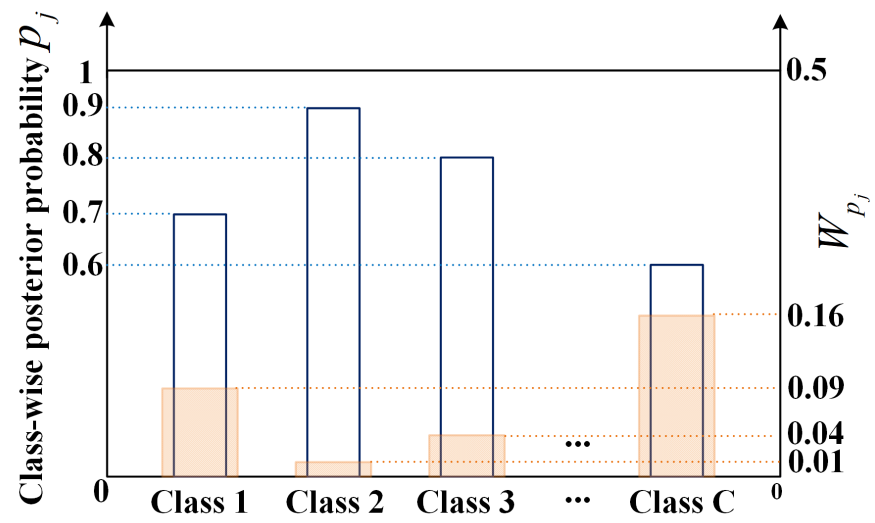

Figure 3: Illustration of class-wise adaptive weights distribution in $\mathcal{L}_{a f}$.

By performing the adaptive focal loss together with two-stage weakly supervised training, we hope that the class imbalance issue in training data, and various difficulty level of AED between multiple target events will be dynamically regulated and focused during model training. Because in Stage 1, the discriminations between those easy-to-classify acoustic events are well-learned with $\mathcal{L}_{s 1}$, and it results in relatively stable teacher and student models. As the training progresses in Stage 2, the adaptive focal loss can automatically down-weight the contribution of easy events during training, and this makes the training rapidly focus the model on difficult-to-classify events. By using the two-stage training, the $\mathcal{L}_{T F D}, \mathcal{L}_{a f}$ and the BCE losses are well integrated to boost the whole weakly supervised system performances.

\subsection{Event-specific post processing (ESP)}

In both audio tagging and acoustic event detection tasks, the frame-level prediction outputs of the model may be non-consecutive, such as, many detection outliers may produce too many extremely short-duration target events occurrence, resulting in inaccurate time-stamps. Therefore, the traditional way is to apply linear or non-linear filters to smooth the prediction outputs. In DCASE Challenges, Median filtering (MF) has proven to be an very effective method in smoothing the noisy outputs of the student model for AED tasks, such as in [6, authors used median filters with the same window size for any sound event class detection.

However, DCASE 2019 Task 4 is a multiple target events detection instead of single one, the subsequent duration of each event in audio clip varies significantly. Conventional median filtering with fixed window size is no longer suitable for this task. Recent works in [43, 31] used group of median filters with adaptive window size by calculating the average duration of events with strong labels on the development set. However, each event duration is not an uniform distribution, using the average duration to optimize the median filtering window 
size may not the optimal. So, we propose to use event-specific MF window size as:

$$
\mathcal{W}_{c}=\left(\frac{1}{N_{c}} \sum_{i=1}^{N_{c}} L_{i}\right) \cdot \eta
$$

where $\mathcal{W}_{c}, c=1,2, \ldots, C$ is the MF window size of class $c, N_{c}$ is the segment index for the inflection point of cumulative distribution of short-to-long sorted segments of the $c$ th class target event. $L_{i}$ is the duration of $i$-th segment of event $c . \quad \eta$ is a scaling factor and set to $1 / 3$ in our experiments. All strong labeled training clips are used to compute $\mathcal{W}_{c}$.

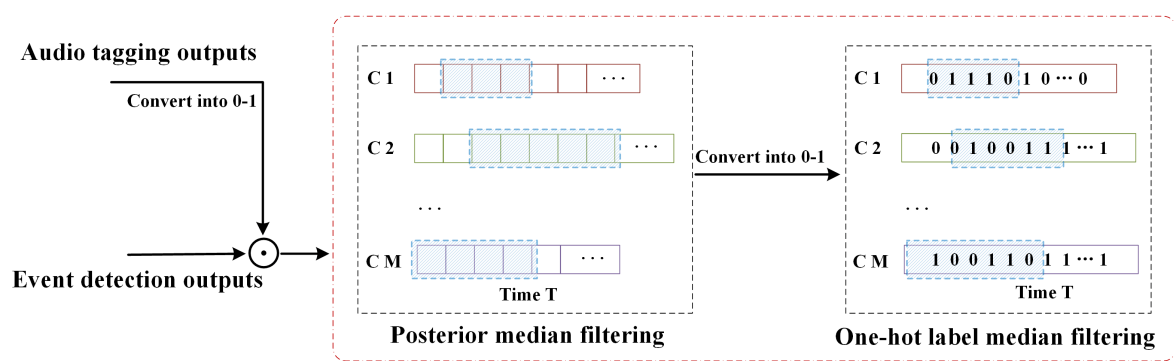

Figure 4: Event-specific post processing.

As shown in Figure 4 we perform the proposed event-specific post processing with Equation (7) on both the frame-level posteriors and one-hot predictions using the same $\mathcal{W}_{c}$. As shown in the bottom right-hand corner of Figure 1. instead of feeding the frame-level acoustic event detection outputs into the proposed ESP directly, here, in Figure 4, we propose to perform an elementwise multiplication between the one-hot audio tagging prediction labels and the frame-level detection posteriors. This operation can not only ensure the consistency of acoustic event detection and audio tagging results, but also can improve the AED performances by integrating the audio tagging guidance.

\section{Experiments and Results}

In this section, we first describe the dataset of DCASE 2019 Task 4 challenge in Section 3.1, then, all the experimental system configurations are presented in Section 3.2 followed by the results and discussions in Section 3.3

\subsection{Dataset}

Our experiments are performed on the dataset of DCASE 2019 Task 4 Challenge [6] ${ }^{1}$. It is a sound event detection task in domestic environments. It aims

\footnotetext{
${ }^{1}$ http://dcase.community/challenge2019/task-sound-event-detection-in-domesticenvironments
} 
to provide not only the event class (AT task), but also the event time boundaries given that multiple events can be present in an audio recording (AED task). The challenge of this task is to explore the possibility to exploit a large amount of unbalanced and unlabeled training data together with a small weakly annotated training set to improve system performance remains but an additional training set with strongly annotated synthetic data is provided.

The dataset for this task is composed of $10 \mathrm{sec}$ audio clips recorded in domestic environment or synthesized to simulate a domestic environment. The focused 10 class sound events are: speech, dog, cat, alarm/bell/ringing, dishes, frying, blender, running water, vacuum cleaner and the electric shaver/toothbrush. The training set includes 1,473 weakly labeled, 13,390 unlabeled and 2,045 strongly labeled audio clips. 1,077 and 692 strongly labeled clips are taken as the development and evaluation clips respectively.

\subsection{System configurations}

We extract $64 \log$ mel-band magnitudes as features, each 10-second clip is transformed into 600 frames. Details of our T-S model structure and its parameters for each block is shown in Figure 1 and section 2 We take both the MT [30] and GL [31] as baselines, because the MT was the official baseline, while GL was the best single system for DCASE 2019 Task 4 and our proposal is improving the GL system. $\alpha=1-\lambda^{e-s}, \beta=e^{-5(1-x)^{2}}$, where $e$ is the current epoch and $x \in[0,1] . \alpha$ is a minimum value, it controls the student model guidance to fine-tune the weight of the teacher model and the consistency cost coefficient $\beta$ was ramped up from 0 to their maximum values, using a sigmoidshaped function. The $\alpha$ and $\beta$ are gradually changing with the model training. More details can reference [29]. The $\gamma, \lambda$ and $s$ is set to 2, 0.996 and 30 respectively in the two-stage training.

$F 1$-score is used to measure the system performances. Not as the traditional recall and precision rates, the $F 1$-score takes into account both the precision and recall statistics of the classification and detection model, and it can be regarded as a harmonic average of the precision and recall. Using $F 1$-score can effectively avoid the impact on the analysis of model performance when the two indicators of precision and recall conflict, and can evaluate the system performance more accurately and conveniently. The acoustic event detection uses an event-based F1 (event-F1) that computed with a $200 \mathrm{~ms}$ collar on onsets and a $200 \mathrm{~ms} / 20 \%$ of the events length collar on offsets, and a segment-based $F 1$ (segment-F1) on 1 second segment is taken as a secondary measure. Segment-based metrics compare system output and reference in short time segments. Active/inactive state for each event class is determined in a fixed length interval that represents a segment. This alleviates issues related to annotator subjectivity in marking onset and offset of sound events. Event-based metrics compare system output and the corresponding reference event by event. The overall (macro-average) $F 1$-score (AT-F1) at clip level is used to measure the audio tagging performances. The metrics are computed using the sed_eval library [44]. 


\subsection{Results and Discussion}

This subsection presents the results and discussions of our proposed framework, including the overall results of all the proposed techniques in section 3.3.1. the analysis of system training parameters tuning of the adaptive focal loss in section 3.3.2, and followed by the statistical analysis, and class-wise performance comparison of the event-specific post processing.

\subsubsection{Overall validation of the proposed methods}

All techniques proposed in this study are examined on DCASE 2019 Task 4 evaluation set. Results are shown in Table1. The 'MT' and 'GL' are the official baseline and top-1 ranked submission single system in this challenge, respectively, we take both of them as our system performance comparison baseline systems. 'PTS' is the backbone T-S model of our proposed framework in Figure 1. without the proposed target-events based deep feature distillation (TFD), two-stage training with adaptive focal loss (AFL) and the event-specific post processing (ESP) techniques. Technical improvements of PTS over GL is presented in section 2.1. 'DFD-MSE' and 'DFD-D' represent performing the deep feature $\mathrm{KD}$ on two transformed feature maps $\mathbf{F}_{t} \cdot \mathbf{W}_{t}, \mathbf{F}_{s} \cdot \mathbf{W}_{s}$ using the conventional MSE criterion [40, and on the frame-level Euclidean distance similarity matrix $D$ respectively. Both of them are performed on all the training data because they do not need any strong-label information. 'DFD-D $(w+u)$ ' means only performing the 'DFD-D' on the weakly and unlabeled training clips.

Table 1: Development/Evaluation set $F 1$-scores (\%) of the proposed methods, the DCASE 2019 Task 4 official baseline (MT) and top-1 ranked single system (GL).

\begin{tabular}{llccc}
\hline ID & System & Event-F1 & Segment-F1 & AT-F1 \\
\hline 0 & MT (Official baseline [30]) & $-/ 25.8$ & $-/ 53.7$ & $-/ 45.8$ \\
1 & GL (Top-1 [31]) & $\mathbf{4 3 . 3 / 4 0 . 5}$ & $\mathbf{6 7 . 1} / \mathbf{6 6 . 5}$ & $\mathbf{7 4 . 1} / \mathbf{7 0 . 2}$ \\
\hline 2 & PTS(w/o BGRUs) & $42.0 / 39.4$ & $63.3 / 61.0$ & $71.2 / 69.1$ \\
3 & PTS & $43.1 / 40.9$ & $69.0 / 68.1$ & $75.0 / 73.4$ \\
4 & PTS+DFD-MSE & $44.1 / 41.3$ & $69.9 / 69.6$ & $71.3 / 68.7$ \\
5 & PTS+DFD-D & $44.4 / 41.7$ & $73.2 / 71.1$ & $73.4 / 72.9$ \\
\hline 6 & PTS+TFD & $48.0 / 45.4$ & $71.7 / 70.2$ & $78.6 / 77.1$ \\
7 & PTS+TFD+DFD-D(w+u) & $42.9 / 40.1$ & $72.9 / 72.0$ & $75.2 / 73.3$ \\
8 & PTS+TFD+AFL & $48.9 / 47.1$ & $75.6 / 74.0$ & $80.0 / 78.1$ \\
9 & PTS+TFD+AFL+ESP & $\mathbf{5 1 . 6} / \mathbf{4 9 . 8}$ & $\mathbf{7 6 . 4} / \mathbf{7 5 . 9}$ & $\mathbf{8 3 . 4} / \mathbf{8 1 . 2}$ \\
\hline
\end{tabular}

In Table 1 , by comparing system 0 and 1 , it is clear that, the best single system GL in DCASE 2019 Task 4 challenge outperforms the official baseline MT model significantly. When we compare system 2 with 1 , it is clear that, the proposed backbone T-S network without BGRUs feature concatenation is much worse than GL system. The independent branch design of AED and AT shows no advantage. However, when we combine the feature representations 
from BGRUs with the standard deep features from CNN Blocks, the performances of proposed T-S model significantly improved, it achieves $0.4 \%, 1.6 \%$ AED and 3.2\% AT F1-score improvements over GL on the real evaluation set. It indicates that, after being combined with the independent design of detection and classification branches, the advantage of complementary information in temporal sequential features from BGRUs are well exploited.

Moreover, when comparing system 6,8 and 9 with system 3, continuous performance gains are obtained by adding the proposed TFD, AFL and ESP techniques into the backbone model, either on the development set or the real evaluation test set. Such as, comparing system 6 with 3, the target-event based deep feature distillation improves the event-F1 from $43.1 \%$ to $48.0 \%$ on development set, and $41.3 \%$ to $45.4 \%$ on evaluation set, the AT-F1 also has more than $3.0 \%$ absolute gains on both test sets. Although the segment-F1 improvements are relatively slight, these performance gains are good to show the effectiveness of the proposed TFD, because as presented in Section 2.2, the principle of TFD is to maximally utilize the strongly labeled target-events frame-level boundary information.

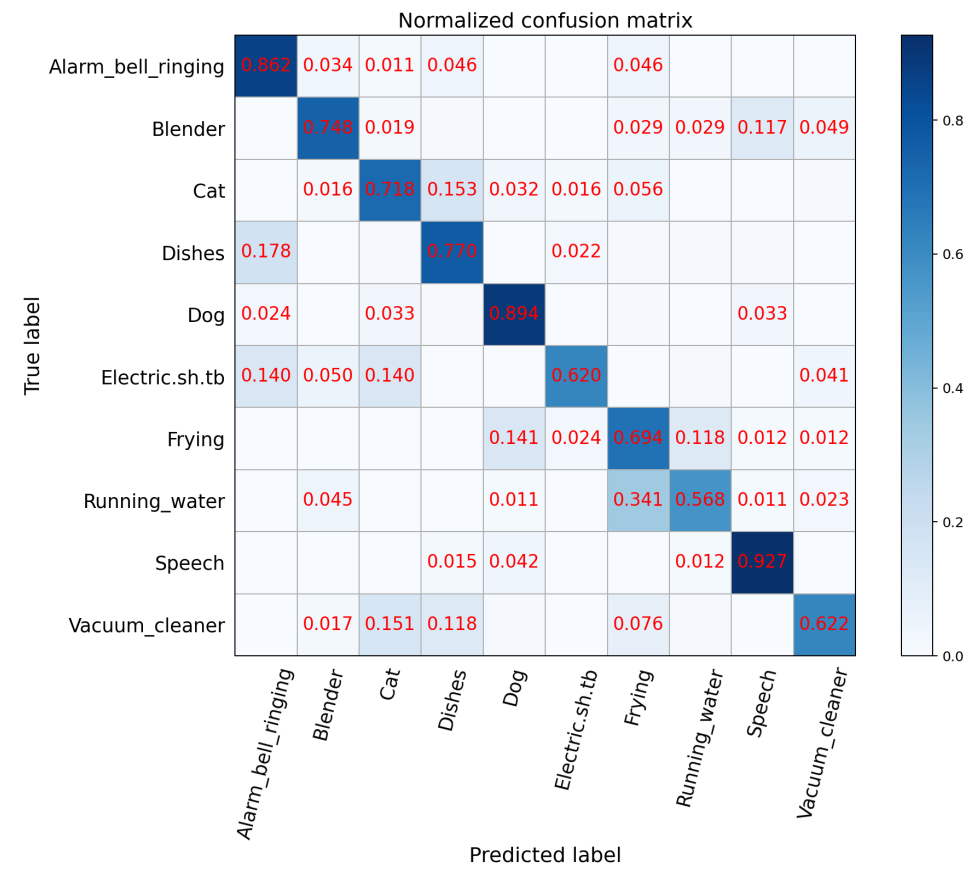

Figure 5: Confusion matrix heat map image of system 2 (the baseline) on the evaluation set for audio tagging.

Comparing system 8 with 6 , we see absolute $0.9 \%$ to $3.9 \%$ F1 performance 


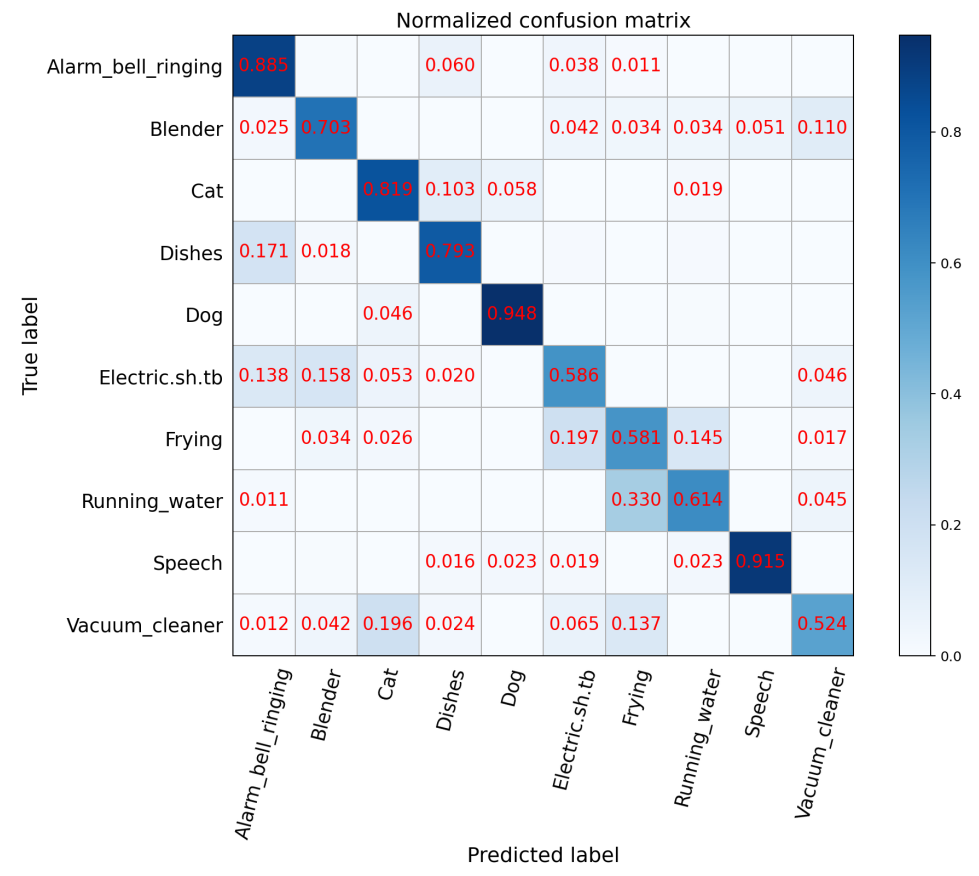

Figure 6: Confusion matrix heat map image of system 9 (the best) on the evaluation set for audio tagging.

gains are achieved on both development and evaluation sets. It means that the proposed two-stage training with adaptive focal loss outperforms the one-stage training strategy significantly, because all the systems from 2 to 7 are trained only using the standard one-stage teacher-student BCE loss as in the GL system. By introducing the adaptive focal loss, we change the system training from standard one-stage to the two-stage way. The consistent performance gains on both development and evaluation set reflect that, the proposed AFL not only enhances the system performances, but also provide more robust and stable acoustic models.

Finally, in system 9, we achieve the best results for both the acoustic event detection and audio tagging tasks. Such as, on the evaluation set, this system outperforms the GL significantly by absolute $9.3 \%, 9.4 \%$ and $11.0 \% F 1$-scores in event-based, segment-based AED and macro-average AT-F1, respectively. We think that these improvements are good enough to prove the effectiveness of our proposed techniques. In addition, when comparing system 9 with 8 , $0.8 \%$ to $3.4 \%$ absolute $\mathrm{F} 1$ improvements are obtained, especially for the AT task ( $>3.0 \%$ gain), it indicates that, the event-specific post processing can smooth the short-duration outliers very well. Furthermore, to show more detail 
classification performances of individual acoustic classes, in Figure 5 and Figure 6. we present the classification confusion matrix of system 9 (the best) and system 2 (baseline system) for AT on the evaluation set as a heat map image. By comparing these two images, it's clear that the AT performance of almost all the events are significantly improved. Moreover, in Figure 6, we note that the dog, speech and alarm bell ringing are much easier to be predicted than other acoustic events; The most difficult classes to predict are the electric shaver toothbrush, frying, and vacuum cleaner, almost $50 \%$ of them are misclassified as other events.

In addition, to further validate the proposed TFD, system 4, 5 are built for performance comparison, we see that there are big performance gaps between system 4, 5 and system 6 , it tells us that the conventional deep feature distillations performed on the whole feature maps are not better than the target-event based one, even they can be trained using all the training data. By comparing system 5 with 4 , we see that performing knowledge distillation using frame-level Euclidean similarity is better than using the conventional MSE. Furthermore, we also try to combine the TFD and DFD-D $(w+u)$ together, it's interesting to find that adding weakly and unlabeled data doesn't bring any additional performance gain as we expected.

\subsubsection{Detail examination of the adaptive focal loss}

Table 2 shows the detail examination results of the proposed adaptive focal loss in section 2.3. The first line results represent the system 6 (PTS+TFD) results in Table 1 without adaptive focal loss (single-stage training, Stage 1), and the setup with Epoch $=15$ and $\gamma=2$ represents the system 8 (PTS+TFD+AFL) in Table 1 that trained using two-stage training strategy with the adaptive focal loss, this setup means that in Stage 2, there are $200-15=185$ epochs are performed. The Epoch $(\tau)$ is the first normal GL training epochs that defined in Stage 1. The total training epoch is fixed to 200 for all the systems.

Table 2: Development/Evaluation set F1-scores (\%) of the system training without (1st line, Stage 1) and with adaptive focal loss.

\begin{tabular}{cc|ccc}
\hline Epoch $(\tau)$ & $\gamma$ & Event-F1 & Segment-F1 & AT-F1 \\
\hline 200 & - & $48.0 / 45.4$ & $71.7 / 70.2$ & $78.6 / 77.1$ \\
\hline 10 & 2 & $\mathbf{4 9 . 1} / 47.0$ & $75.4 / \mathbf{7 5 . 1}$ & $78.0 / 76.6$ \\
15 & 2 & $48.9 / \mathbf{4 7 . 1}$ & $\mathbf{7 5 . 6} / 74.0$ & $\mathbf{8 0 . 0} / \mathbf{7 8 . 1}$ \\
30 & 2 & $48.0 / 47.1$ & $70.8 / 70.1$ & $76.9 / 76.4$ \\
15 & 1 & $45.5 / 44.8$ & $74.3 / 73.3$ & $75.3 / 74.4$ \\
15 & 3 & $48.0 / 46.6$ & $75.6 / 74.0$ & $77.0 / 75.2$ \\
\hline
\end{tabular}

From Table 2, we see that only 15 epoches GL training is enough to produce a relatively stable T-S model. During these epoches, those easy acoustic events are already well-trained. After this iteration, in Stage 2, the model will be guided to focus on the difficult-to-classify events by the introduced adaptive focal loss in 
next training iterations. In addition, it's clear that the focal loss is very sensitive to the scaling factor $\gamma$ of Equation (4), because it controls the loss contribution of each training clips for the total loss. It is a very important parameter, too large or too small will make the model produce different deviation. Therefore, the relatively best setup with $\tau=15, \gamma=2$ is taken to train our final systems, such as system 8 and 9 in Table 1. In addition, it can be seen from Table 2 that the results in the first row (single-stage training mode) of the performance comparison of all systems using the two-stage training mode based on AFL are improved in the segment based F1-score. At the same time, it can be seen that the impact of entering the second stage in different epochs on the system is also very obvious. Too early or too late will bring different performance.

\subsubsection{Validation of event-specific post processing}
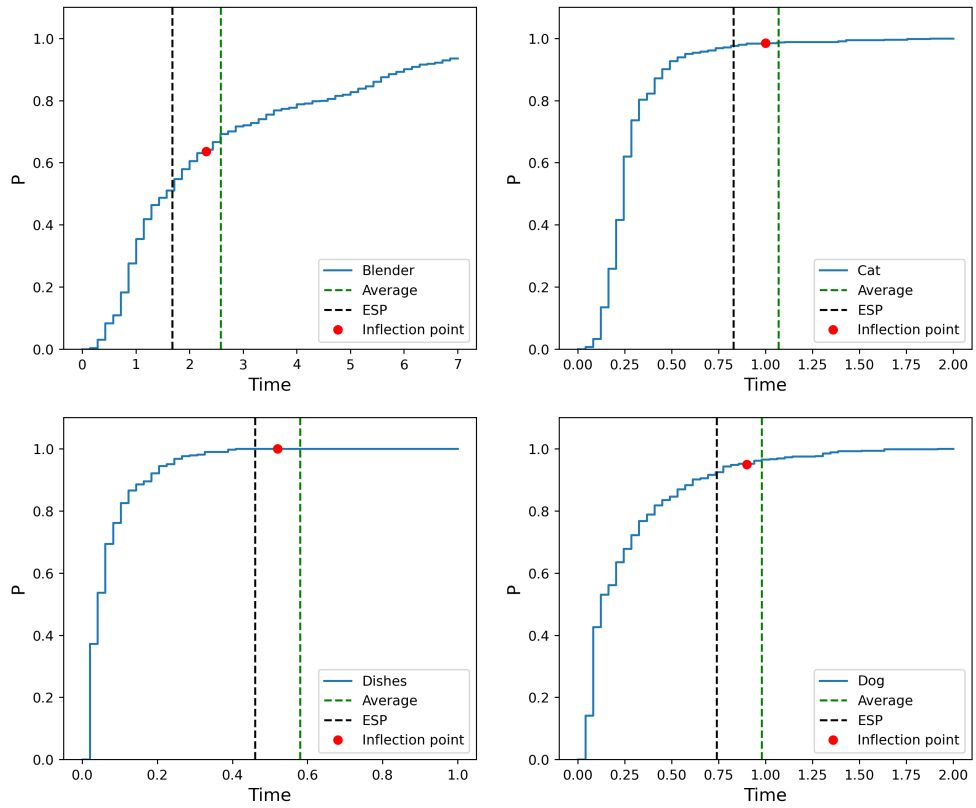

Figure 7: Illustration of cumulative distribution of event-based duration that calculated from strong-labels for four different acoustic events: Blender, Cat, Dishes and Dog.

Figure 7 illustrates four acoustic types' cumulative distributions of eventbased duration that calculated from strong-labels. The x-axis (abscissa axis) is the time-duration of each event, and the y-axis (ordinate axis) is the normalized occupation probability. As shown in Equation (7), the inflection point of this cumulative distribution is used to compute the ESP duration for each target 
event. Comparing the four subfigures, we see that there is a big duration gap for one event when it occurs in different clips. From each subfigure, we can see the average duration (green line) of each event deviates far from the proposed ESP duration (black line) that computed according to the event-based cumulative distributions in Equation (7). Furthermore, from the four example distributions, and indicated by the distribution changing of y-axis and the red inflection points, it's clear that the durations of most clips are more closer to the ESP durations than the average ones. Meanwhile, the long flat-tails of these distributions vary with $\mathrm{x}$-axis also indicates that, there are obvious differences in the MF window size calculation by our proposed ESP and the traditional average way. Therefore, we argue that the proposed ESP duration is better to reflect the real distribution than the average one that used in most previous works 43, 31, to perform the median filtering post processing.

Table 3: Class-wise performances $(F 1$-score $(\%))$ on the evaluation set with different postprocessing methods. Bold fonts are used to highlight the $F 1$-scores with the largest performance improvements with respect to Avg and ESP.

\begin{tabular}{ccc}
\hline Event class & Avg(AED / AT) & ESP (AED / AT) \\
\hline Alarm/bell/ringing & $47.6 / \mathbf{7 7 . 4}$ & $48.3 / \mathbf{8 3 . 3}$ \\
Blender & $39.5 / 63.7$ & $40.3 / 72.3$ \\
Cat & $\mathbf{5 7 . 1} / 86.1$ & $\mathbf{6 6 . 2} / 89.6$ \\
Dishes & $34.5 / 74.6$ & $38.5 / 78.2$ \\
Dog & $52.2 / 87.7$ & $52.8 / 88.3$ \\
Electric shaver/toothbrush & $43.5 / 78.0$ & $51.4 / 80.8$ \\
Frying & $51.5 / 73.8$ & $54.5 / 80.2$ \\
Running water & $32.9 / 68.1$ & $31.2 / 63.2$ \\
Speech & $56.9 / 93.4$ & $58.9 / 93.3$ \\
Vacuum cleaner & $54.7 / 77.7$ & $55.2 / 82.3$ \\
\hline Overall & $47.1 / 78.0$ & $49.8 / 81.2$ \\
\hline
\end{tabular}

Table 3 demonstrates the class-wise performances with different median filtering window size as the predictions post processing. The 'Avg' represents using adaptive median filtering window size with average duration of each event class. 'ESP' is our proposed event-specific post processing. 'AED/AT' is the event-based and class-wise $F 1$-score for acoustic event detection and audio tagging. It's clear to see that, the proposed ESP is very effective to improve the system performances for most target events, especially for those short-duration ones as the blender, cat and dishes, etc. Such as, for the blender class, the AT-F1 is significantly improved from $63.7 \%$ to $72.3 \%$, and for the cat class, the event-based AED F1-score is improved from $57.1 \%$ to $66.2 \%$. Compared with the 'Avg' based median filtering, the proposed ESP improves the overall AED/AT F1-score from $47.1 / 78.0 \%$ to $49.8 / 81.2 \%$.

In addition, in Table 3 it is worth noting that, on the real evaluation set, the class-wise F1-scores of all the event classes except for running water have 
been improved. This may due to the fact that, the MF window size of EPS is calculated according to the strongly labeled data of 10 event classes, however, the available strongly labeled data is very limited, and there is heavy data imbalance between different classes. For class 'running water', the strongly labeled data amount is much less than that of other classes. Therefore, the MF window size of EPS calculated for this class may have a certain deviation from the actual length distribution of the running water events, which makes the proposed ESP for the running water prediction smoothing fails to outperform the standard 'Avg'.

\section{Conclusion}

In this work, we propose a new joint framework for weakly supervised acoustic event detection and audio tagging. Different from previous works that also based on the teacher-student learning and CRNN frameworks, our proposal learns the models for acoustic event detection and audio tagging tasks as two independent branches in the whole architecture. Three new methods are proposed to improve the joint framework, including a frame-level target-events based deep feature distillation, a two-stage training strategy with adaptive focal loss, and an event-specific post processing. All of these techniques are validated on the dataset of DCASE 2019 Task 4 challenge, experimental results show that the new joint framework with all the proposed techniques achieves competitive performances in both acoustic event detection and audio tagging tasks. On the real evaluation set of DCASE 2019 Task 4 challenge, our proposed system significantly outperforms the best single submission system (GL) by absolute $9.3 \%, 9.4 \%$ and $11.0 \%$ F1-scores in event-based, segment-based AED and macro-average AT-F1, respectively. Our future work will focus on generalizing the proposed framework to other acoustic event detection tasks, try our separate models for AT and AED tasks with all the proposed techniques, and improving the event-specific post processing to fix the strongly labeled data imbalance issue.

\section{Acknowledgment}

This work was funded by the National Natural Science Foundation of China (Grant No.62071302).

\section{References}

[1] C. Debes, A. Merentitis, S. Sukhanov, M. Niessen, N. Frangiadakis, A. Bauer, Monitoring activities of daily living in smart homes: Understanding human behavior, IEEE Signal Processing Magazine 33 (2) (2016) $81-94$. 
[2] Y. Zigel, D. Litvak, I. Gannot, A method for automatic fall detection of elderly people using floor vibrations and sound-proof of concept on human mimicking doll falls, IEEE transactions on biomedical engineering 56 (12) (2009) 2858-2867.

[3] E. Wold, T. Blum, D. Keislar, J. Wheaten, Content-based classification, search, and retrieval of audio, IEEE multimedia 3 (3) (1996) 27-36.

[4] Q. Jin, P. Schulam, S. Rawat, S. Burger, D. Ding, F. Metze, Event-based video retrieval using audio, in: Proceedings of the 13th Annual Conference of the International Speech Communication Association (Interspeech), ISCA, 2012, pp. 2085-2088.

[5] R. Serizel, N. Turpault, H. Eghbal-Zadeh, A. P. Shah, Large-scale weakly labeled semi-supervised sound event detection in domestic environments, in: Proceedings of the Detection and Classification of Acoustic Scenes and Events 2018 Workshop (DCASE 2018), 2018.

[6] N. Turpault, R. Serizel, J. Salamon, A. P. Shah, Sound event detection in domestic environments with weakly labeled data and soundscape synthesis, in: Proceedings of the Detection and Classification of Acoustic Scenes and Events 2019 Workshop (DCASE 2019), 2019, pp. 253-257.

[7] N. Turpault, S. Wisdom, H. Erdogan, J. Hershey, R. Serizel, E. Fonseca, P. Seetharaman, J. Salamon, Improving sound event detection in domestic environments using sound separation, arXiv preprint, arXiv:2007.03932.

[8] R. Serizel, N. Turpault, Sound event detection from partially annotated data: Trends and challenges, in: Proc. IcETRAN conference, 2019.

[9] A. Shah, A. Kumar, A. G. Hauptmann, B. Raj, A closer look at weak label learning for audio events, arXiv preprint, arXiv:1804.09288.

[10] B. McFee, J. Salamon, J. P. Bello, Adaptive pooling operators for weakly labeled sound event detection, IEEE/ACM Transactions on Audio, Speech, and Language Processing 26 (11) (2018) 2180-2193.

[11] Y. Huang, L. Lin, S. Ma, X. Wang, H. Liu, Y. Qian, M. Liu, K. Ouch, Guided multi-branch learning systems for DCASE 2020 task 4, arXiv preprint, arXiv:2007.10638.

[12] I. McLoughlin, H. Zhang, Z. Xie, Y. Song, W. Xiao, Robust sound event classification using deep neural networks, IEEE/ACM Transactions on Audio, Speech, and Language Processing 23 (3) (2015) 540-552.

[13] S. Hong, Y. Zou, W. Wang, Gated Multi-Head Attention Pooling for Weakly Labelled Audio Tagging, in: Proceedings of the 21th Annual Conference of the International Speech Communication Association (Interspeech), 2020, pp. 816-820. 
[14] C.-C. Kao, B. Shi, M. Sun, C. Wang, A Joint Framework for Audio Tagging and Weakly Supervised Acoustic Event Detection Using DenseNet with Global Average Pooling, in: Proceedings of the 21th Annual Conference of the International Speech Communication Association (Interspeech), 2020, pp. $846-850$.

[15] I. Park, H. K. Kim, Two-Stage Polyphonic Sound Event Detection Based on Faster R-CNN-LSTM with Multi-Token Connectionist Temporal Classification, in: Proceedings of the 21th Annual Conference of the International Speech Communication Association (Interspeech), 2020, pp. 856-860.

[16] J. Zhang, W. Ding, J. Kang, L. He, Multi-Scale Time-Frequency Attention for Acoustic Event Detection, in: Proceedings of the 20th Annual Conference of the International Speech Communication Association (Interspeech), 2019, pp. 3855-3859.

[17] D. de Benito-Gorron, S. Segovia, D. Ramos, D. T. Toledano, MultiResolution Mean Teacher For DCASE 2020 Task 4, Tech. rep. (2020).

[18] X. Lu, P. Shen, S. Li, Y. Tsao, H. Kawai, Class-Wise Centroid Distance Metric Learning for Acoustic Event Detection, in: Proceedings of the 20th Annual Conference of the International Speech Communication Association (Interspeech), 2019, pp. 3614-3618.

[19] X. Li, V. Chebiyyam, K. Kirchhoff, Multi-Stream Network with Temporal Attention for Environmental Sound Classification, in: Proceedings of the 20th Annual Conference of the International Speech Communication Association (Interspeech), 2019, pp. 3604-3608.

[20] H. Dinkel, K. Yu, Duration robust weakly supervised sound event detection, in: Proceedings of the 45th International Conference on Acoustics, Speech, and Signal Processing (ICASSP), IEEE, 2020, pp. 311-315.

[21] K. Miyazaki, T. Komatsu, T. Hayashi, S. Watanabe, T. Toda, K. Takeda, Weakly-supervised sound event detection with self-attention, in: Proceedings of the 45th International Conference on Acoustics, Speech, and Signal Processing (ICASSP), IEEE, 2020, pp. 66-70.

[22] X. Li, V. Chebiyyam, K. Kirchhoff, Multi-stream network with temporal attention for environmental sound classification, arXiv preprint, arXiv:1901.08608.

[23] K. Lu, C.-S. Foo, K. K. Teh, H. D. Tran, V. R. Chandrasekhar, Semisupervised audio classification with consistency-based regularization., in: Proceedings of the 20th Annual Conference of the International Speech Communication Association (Interspeech), 2019, pp. 3654-3658.

[24] H. Zhang, M. Cisse, Y. N. Dauphin, D. Lopez-Paz, mixup: Beyond empirical risk minimization, in: International Conference on Learning Representations, 2017. 
[25] V. Verma, A. Lamb, J. Kannala, Y. Bengio, D. Lopez-Paz, Interpolation consistency training for semi-supervised learning, arXiv preprint, arXiv:1903.03825.

[26] H. L. Bear, I. Nolasco, E. Benetos, Towards joint sound scene and polyphonic sound event recognition, arXiv preprint, arXiv:1904.10408.

[27] C. C. Chatterjee, M. Mulimani, S. G. Koolagudi, Polyphonic sound event detection using transposed convolutional recurrent neural network, in: Proceedings of the 45th International Conference on Acoustics, Speech, and Signal Processing (ICASSP), IEEE, 2020, pp. 661-665.

[28] R. Serizel, N. Turpault, H. Eghbal-Zadeh, A. P. Shah, Large-scale weakly labeled semi-supervised sound event detection in domestic environments, in: Proceedings of the Detection and Classification of Acoustic Scenes and Events 2018 Workshop (DCASE 2018), 2018, pp. 19-23.

[29] A. Tarvainen, H. Valpola, Mean teachers are better role models: Weightaveraged consistency targets improve semi-supervised deep learning results, in: Proceedings of the 31st International Conference on Neural Information Processing Systems (NIPS'17), 2017, pp. 1195-1204.

[30] L. JiaKai, Mean teacher convolution system for DCASE 2018 task 4, in: Proceedings of the Detection and Classification of Acoustic Scenes and Events 2018 Workshop (DCASE 2018), 2018.

[31] L. Lin, X. Wang, H. Liu, Y. Qian, Guided learning for weakly-labeled semisupervised sound event detection, in: Proceedings of the 45th International Conference on Acoustics, Speech, and Signal Processing (ICASSP), IEEE, 2020, pp. 626-630.

[32] Y. Huang, X. Wang, L. Lin, H. Liu, Y. Qian, Multi-branch learning for weakly-labeled sound event detection, in: Proceedings of the 45th International Conference on Acoustics, Speech, and Signal Processing (ICASSP), IEEE, 2020, pp. 641-645.

[33] R. Shi, R. W. Ng, P. Swietojanski, Teacher-student training for acoustic event detection using audioset, in: Proceedings of the 44th International Conference on Acoustics, Speech, and Signal Processing (ICASSP), IEEE, 2019, pp. 875-879.

[34] A. Romero, N. Ballas, S. E. Kahou, A. Chassang, C. Gatta, Y. Bengio, Fitnets: Hints for thin deep nets, arXiv preprint, arXiv:1412.6550.

[35] G. Hinton, O. Vinyals, J. Dean, Distilling the knowledge in a neural network, arXiv preprint, arXiv:1503.02531.

[36] A. Pilzer, S. Lathuiliere, N. Sebe, E. Ricci, Refine and distill: Exploiting cycle-inconsistency and knowledge distillation for unsupervised monocular depth estimation, in: Proceedings of the IEEE Conference on Computer Vision and Pattern Recognition(CVPR), 2019, pp. 9768-9777. 
[37] X. Cheng, Z. Rao, Y. Chen, Q. Zhang, Explaining knowledge distillation by quantifying the knowledge, in: 2020 IEEE/CVF Conference on Computer Vision and Pattern Recognition (CVPR), pp. 12925-12935.

[38] S. I. Mirzadeh, M. Farajtabar, A. Li, H. Ghasemzadeh, Improved Knowledge Distillation via Teacher Assistant: Bridging the Gap Between Student and Teacher.

[39] Y. Dong, H. Su, J. Zhu, B. Zhang, Improving interpretability of deep neural networks with semantic information, in: 2017 IEEE Conference on Computer Vision and Pattern Recognition (CVPR), pp. 975-983.

[40] C.-C. Chang, C.-C. Kao, M. Sun, C. Wang, Intra-utterance similarity preserving knowledge distillation for audio tagging, in: Proceedings of the 21th Annual Conference of the International Speech Communication Association (Interspeech), ISCA, 2020, pp. 851-855.

[41] T.-Y. Lin, P. Goyal, R. Girshick, K. He, P. Dollár, Focal loss for dense object detection, in: Proceedings of the IEEE International Conference on Computer Vision (ICCV), 2017, pp. 2980-2988.

[42] Y. Bengio, J. Louradour, R. Collobert, J. Weston, Curriculum learning, in: Proceedings of the 26th Annual International Conference on Machine Learning(ICML), 2009, pp. 41-48.

[43] L. Delphin-Poulat, C. Plapous, Mean teacher with data augmentation for DCASE 2019 task 4, in: Proceedings of the Detection and Classification of Acoustic Scenes and Events 2019 Workshop (DCASE 2019), 2019.

[44] A. Mesaros, T. Heittola, T. Virtanen, Metrics for polyphonic sound event detection, Applied Sciences 6 (162). 